\title{
IMPLANTAÇÃO DE UM MINI-PACS (SISTEMA DE ARQUIVAMENTO E DISTRIBUIÇÃO DE IMAGENS) EM HOSPITAL UNIVERSITÁRI0*
}

\author{
Paulo Mazzoncini de Azevedo-Marques ${ }^{1}$, Clóvis SimãoTrad ${ }^{2}$, Jorge Elias Júnior ${ }^{3}$, Antonio Carlos \\ Santos ${ }^{4}$
}

\begin{abstract}
Resumo Este trabalho apresenta a implementação de um mini-PACS (sistema de arquivamento e comunicação de imagens) que está sendo estruturado junto ao Serviço de Radiodiagnóstico do Hospital das Clínicas da Faculdade de Medicina de Ribeirão Preto da Universidade de São Paulo, como parte do projeto de um serviço de radiologia digital (“filmless").
\end{abstract}

Unitermos: Radiologia digital. Sistema de arquivamento e distribuição de imagens. PACS. Radiologia sem filme.

\begin{abstract}
Mini-PACS (picture archiving and communication system) implementation at a university hospital.
This paper describes the implementation of a mini-PACS (picture archiving and communication system) at a university hospital (“Hospital das Clínicas da Faculdade de Medicina de Ribeirão Preto da Universidade de São Paulo"), as a component of a project for a filmless radiology facility.

Key words: Digital radiology. Picture archiving and communication system. PACS. Filmless radiology.
\end{abstract}

\section{INTRODUÇÃ̃O}

O expressivo progresso da radiologia nas últimas décadas, associado ao aparecimento e aperfeiçoamento de novos métodos de imagem como o ultra-som e a ressonância magnética, por exemplo, fizeram do diagnóstico por imagem uma das mais excitantes áreas da medicina. Seu impacto é tão grande que a abordagem diagnóstica médica vem sofrendo sua influência e sendo bastante modificada. Dentre as várias modificações, a implantação de sistemas de armazenamento e comunicação de imagens ("picture archiving and communication system"-PACS) é provavelmente a de maior impacto. O PACS (Figura 1) é um sistema de arquivamento e comunicação voltado

* Trabalho realizado no Serviço de Radiodiagnóstico do Hospital das Clínicas da Faculdade de Medicina de Ribeirão Preto da Universidade de São Paulo (FMRP-USP), Ribeirão Preto, SP.

1. Engenheiro Eletrônico, Doutor em Física Aplicada, Docente do Centro de Ciências das Imagens e Física Médica da FMRP-USP.

2. Médico Radiologista, Doutor em Clínica Médica, Docente e Coordenador do Centro de Ciências das Imagens e Física Médica da FMRP-USP.

3. Médico Radiologista, Mestre em Neurologia, Docente do Centro de Ciências das Imagens e Física Médica da FMRP USP.

4. Médico Radiologista, Doutor em Neurologia, Docente do Centro de Ciências das Imagens e Física Médica da FMRPUSP.

Endereço para correspondência: Prof. Dr. Paulo M. de Azevedo-Marques. Rua Ceará, 3005, apto. 22, Jardim Paulistano. Ribeirão Preto, SP, 14090-300.E-mail: pmarques@ fmrp.usp.br

Aceito para publicação em 30/5/2001. para o diagnóstico por imagem que permite o pronto acesso, em qualquer setor do hospital ou clínica, de imagens médicas em formato digital, sendo caracterizado por quatro subsistemas: aquisição, exibição, disponibilização e armazenamento de imagens.

\section{Aquisição de imagens}

Apesar do aumento no uso de modalidades de imagem que permitem a realização de cortes seccionais, tais como a tomografia computadorizada (TC), o ultra-som (US) e a ressonância magnética (RM), as quais, de modo geral, fornecem imagens em formato digital, os exames de radiologia convencional continuam representando $70 \%$ dos exames realizados em um departamento de radiologia ${ }^{(1)}$

Para esse tipo de procedimento existem, basicamente, duas formas de se fazer a aquisição digital de imagens. Uma, é a utilização de sistemas convencionais tela/filme e a posterior digitalização da imagem por intermédio de um "scanner". Existem muitos tipos de digitalizadores de filmes, incluindo sistemas com CCD ("charge-coupled devices") e varredura por feixe de "laser". Digitalizadores de filmes podem ser introduzidos sem grandes alterações na rotina do serviço, possibilitando uma transição suave para um sistema baseado em imagem digital. Porém, existem limitações importantes nessa solução: com esse sistema não há re- dução de tempo ou trabalho, continuam existindo problemas de sobre ou subexposição, e acrescenta-se ainda maior risco de erros associados à digitalização. A outra solução seria a utilização de sistemas de radiografia computadorizada ("computed radiography" - CR), introduzidos em 1983 pela Companhia Fuji (Kanagawa, Japão) ${ }^{(2)}$, que oferecem uma alternativa ao uso dos sistemas tela/filme e "scanner". Nesses sistemas as imagens digitais são diretamente produzidas em uma placa de imagem à base de fósforo ("imaging plate"), podendo, na seqüência, serem visibilizadas em monitores ou convertidas para imagem analógica em filme por meio de uma processadora "laser". Os sistemas CR são compatíveis com a maioria dos sistemas de raios- $X$ fixos e portáteis, possuindo latitude de exposição bastante larga. Isso resulta em imagens com densidade adequada em uma faixa de níveis de exposição ampla, eliminando os problemas de sobreexposição e de subexposição das imagens.

\section{Disponibilização de imagens}

A disponibilização de imagens refere-se ao problema de mover uma imagem e seus dados associados de uma localização para outra. Em um PACS, um sistema de disponibilização de imagens é necessário para transferir as imagens do local de aquisição, ou do sistema de armazenamento, para a estação de visibilização. 


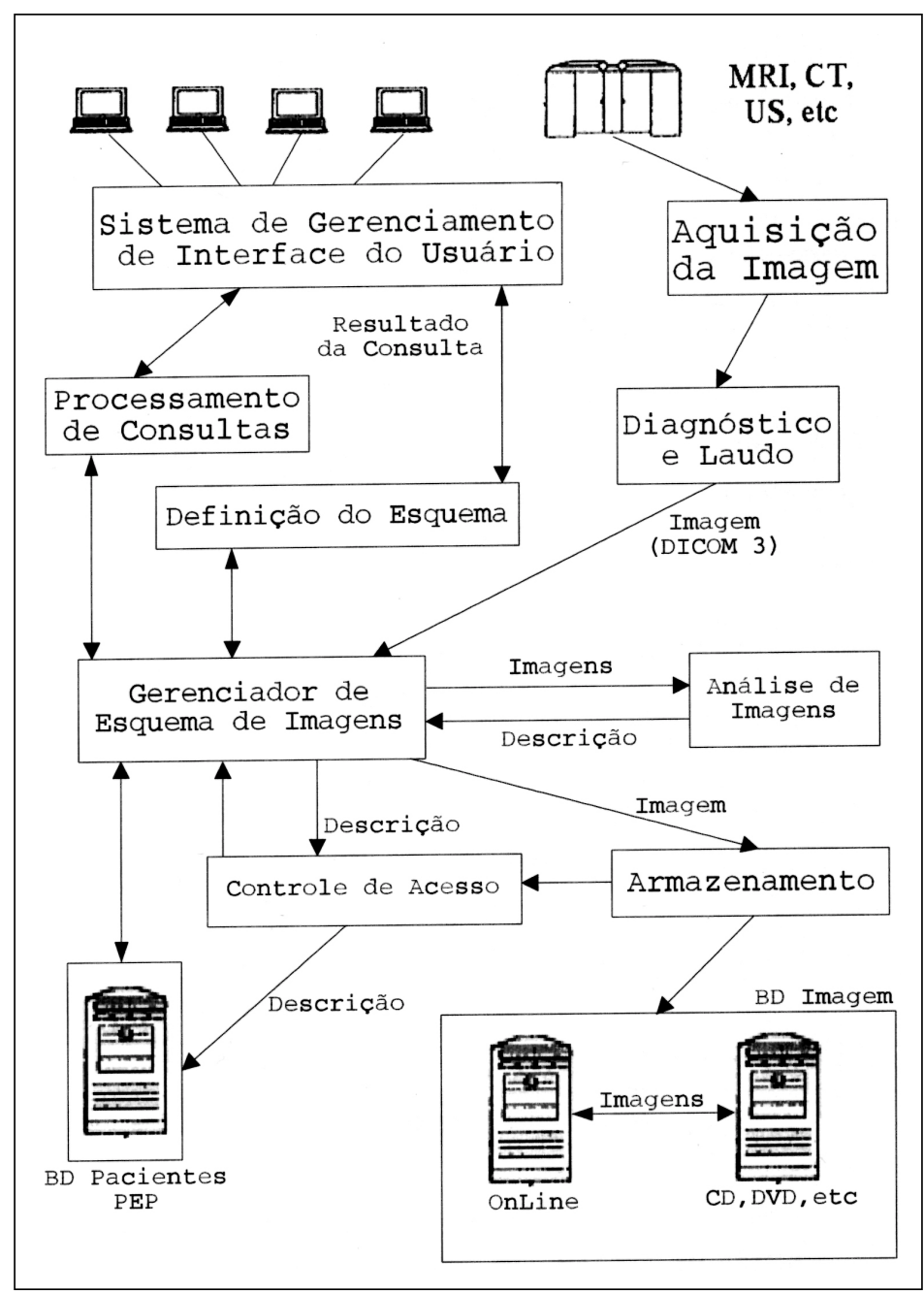

Figura 1. Esquema genérico de uma rede PACS.

Em muitos dos primeiros sistemas de imagem baseados em computador (TC e RM, por exemplo), o problema da distribuição de imagens não era abordado ou estava atrelado a soluções proprietárias dos fabricantes. Alternativas incluíam a transferência física de gravações ou a conexão dos sistemas de exibição ao computador central do sistema. Com a implantação generalizada de redes de computadores ficou aparente a necessidade de uma metodologia uniforme para transferência de informações de imagens.

No meio da década de 70 , um comitê coordenado pelo Colégio Americano de Radiologia ("American College of Radiology" - ACR) e pela Associação Nacional de Fabricantes de Equipamentos Elétricos ("National Electrical Manufactures' Association" - NEMA) foi formado para trabalhar na padronização de programas e equipamentos para acesso e distribuição de imagens médicas. Recentemente, foi adotada como padrão a versão 3.0 do modelo ACR-NEMA, normalmente conhecida como DICOM ("digital imaging and communication in medicine"). A maioria dos fabricantes, senão todos, de dispositivos para obtenção de imagem em formato digital está trabalhando no desenvolvimento de produtos que sejam DICOM compatíveis.

\section{Exibição de imagens}

Para muitos usuários, o sistema de exibição, ou "workstation", é o único componente do PACS com o qual irá interagir diretamente. A estação de trabalho deve ser capaz de recuperar imagens de forma rápida e fácil e possibilitar uma navegação intuitiva na base de dados, para facilitar a recuperação e comparação de dados relevantes relacionados ao exame. A definição de uma configuração ideal é bastante complicada, porém existem alguns requisitos mínimos que devem ser respeitados em se tratando de uma estação para interpretação de exames: possuir pelo menos dois monitores de alto brilho, com $1.024 \times 1.024$ pixels no mínimo, apresentar a base de dados de forma otimizada, o tempo de espera entre a seleção da imagem e a sua exibição deve ser bastante curto $(\leq 5 \mathrm{~s})$, possuir programas básicos de processamento de imagem (ajuste de brilho e contraste, "zoom", inversão de escala de cinza, rotação, rolagem e inversão da imagem).

\section{Armazenamento de imagens}

Armazenar é a função de reter as imagens até que elas sejam requisitadas para visibilização. $\mathrm{O}$ armazenamento pode ser dividido em duas áreas distintas: armazenamento rápido ("short-term"), o qual inclui armazenamento (magnético) local, e armazenamento longo ("longterm"), o qual envolve meios de armazenamento óticos, entre outros.

$\mathrm{O}$ armazenamento local (ou magnético), que geralmente refere-se ao disco rígido (HD) do computador, armazena as informações da imagem digital em um disco magnético para uma recuperação rápida. Em geral, as imagens são mantidas armazenadas nesse meio por volta de uma semana, devido ao grande volume de dados e o alto custo dos sistemas magnéticos de alta capacidade. Uma imagem armazenada neste sistema pode geralmente ser acessada em menos de cinco segundos. O armazenamento local é utilizado tanto nas estações de visibilização como nos servidores do sistema.

O armazenamento por longo prazo refere-se geralmente a discos óticos nãoregraváveis, podendo chegar a capacidades de 10 GB cada, discos ótico-magnéticos regraváveis, que permitem armazenar as informações por um período de tempo específico e então apagá-las, arquivos em fita magnética, em CD-ROMs $\mathrm{e}$, mais recentemente, em DVDs.

Embora o PACS venha se tornando rapidamente uma opção tecnológica pre- 
ferida para as tarefas de transmissão, armazenamento, recuperação, visibilização e interpretação de grandes volumes de dados, a implantação de uma rede PACS completa é bastante cara e complexa, sendo necessário o desenvolvimento de um planejamento bem organizado e inteligente para sua execução. Existem muitos artigos na literatura especializada apresentando soluções e estratégias para a implantação de redes PACS em hospitais e serviços de saúde ${ }^{(3-15)}$, e outros discutindo os componentes e interfaces dos sistemas ${ }^{(\mathbf{1 6 - 2 2})}$. Esses artigos apresentam, no geral, soluções baseadas em componentes comerciais em conjunto com componentes desenvolvidos, ou customizados, por equipes locais, sendo implantadas modularmente de modo a tentar minimizar impactos e gastos, possibilitando a aquisição de conhecimentos que deverão ser utilizados no planejamento e implementação das etapas posteriores.

\section{MATERIAL E MÉTODOS}

O Centro de Ciências das Imagens e Física Médica (CCIFM) do Departamento de Clínica Médica da Faculdade de Medicina de Ribeirão Preto (FMRP) vem trabalhando no sentido de iniciar o processo de transição do Serviço de Radiodiagnóstico do Hospital das Clínicas da FMRP para a radiologia "filmless". Radiologia "filmless" refere-se a um hospital, com um ambiente de rede amplo e integrado, no qual o filme foi completamente ou em grande parte substituído por sistemas eletrônicos que adquirem, arquivam, disponibilizam e exibem imagens. Segundo a literatura especializada, a maioria dos serviços médicos farão a transição para a radiologia "filmless" nos próximos dez a vinte anos ${ }^{(4)}$. No Hospital das Clínicas de Ribeirão Preto essa transição tem envolvido a conexão de equipamentos em rede (como TC e RM), o armazenamento de imagens em meio eletrônico (CD-ROM) para posterior disponibilização e o desenvolvimento e implantação de um sistema de informação em radiologia ("radiology information system" - RIS) (23). Atualmente estão conectados em rede dois equipamentos de TC (um Tomoscan Philips e um Somaton
Siemens) e um equipamento de RM (Magneton Vision Siemens). Esses aparelhos estão ligados a um servidor Linux que gerencia a distribuição das imagens por meio de FTP ("file transfer protocol"), utilizando rede "internet" e protocolo TCP/IP ("transmission control protocol/ internet protocol"). As imagens ficam armazenadas em um "disk-array" (DataForce 300/50) para consulta "on-line" e gravadas em CD-ROM para consulta "offline" (Figura 2).

\section{RESULTADOS}

Os equipamentos Siemens (Magneton Vision e Somaton) são DICOM 3 compatíveis e estão conectados em rede por intermédio de um aplicativo da Siemens para gerenciamento de imagens (Magic View 300). Este aplicativo permite que um computador tipo PC (com sistema operacional Windows NT) funcione co- mo um console auxiliar, podendo manipular, arquivar, gravar em CD-ROM e disponibilizar suas imagens através do servidor. As imagens são armazenadas preferencialmente em formato DICOM 3, porém existe também a opção de armazenamento em formato "tiff".

$\mathrm{O}$ equipamento Philips, por ser mais antigo, não é DICOM 3 compatível e está conectado em rede por meio de um sistema de captura de imagens da Medical Systems $^{\mathrm{TM}}$ (ImageExplorer). Este sistema permite capturar as imagens presentes no monitor do equipamento, por intermédio de uma placa de captura de vídeo instalada em um computador do tipo PC (com sistema operacional Windows 98), possibilitando sua manipulação, arquivamento, gravação em CD-ROM e disponibilização através do servidor. Neste caso, as imagens são armazenadas em formatos comerciais para arquivos de imagem (jpeg, bmp, tiff, gif, pcx, etc.).

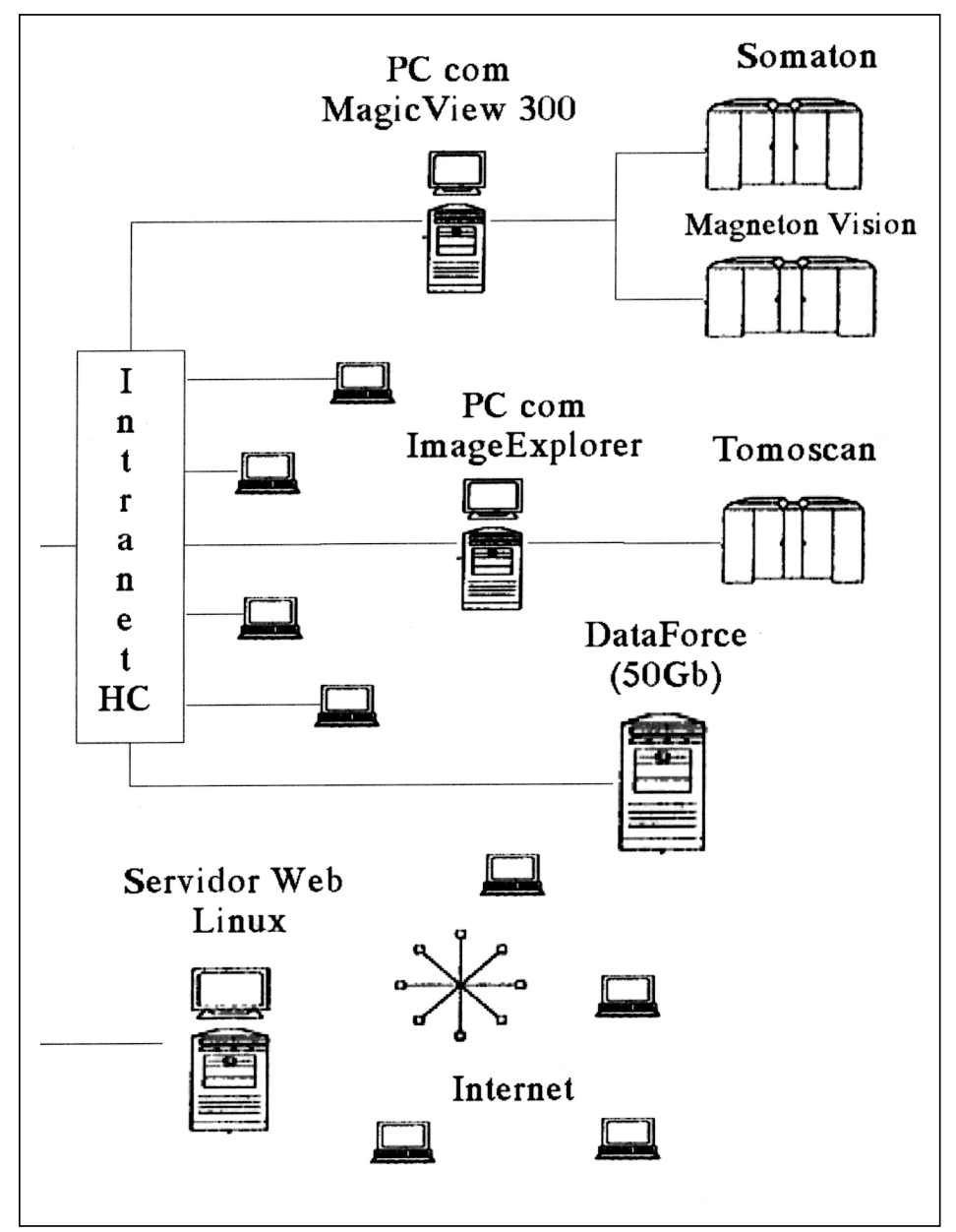

Figura 2. Esquema atual do mini-PACS do Hospital das Clínicas da FMRP-USP. 
O "disk-array" trabalha com sistema operacional Unix, possibilitando gerenciamento e acesso concomitante de até 100 usuários cadastrados, e área para acesso anônimo. Sua capacidade atual de arquivamento é de $50 \mathrm{~GB}$, o que permite disponibilizar para consulta "on-line" um volume de imagens equivalente a um período aproximado de dois meses. Usuários conectados à rede interna do Hospital podem acessar essas imagens diretamente, sem passar pelo servidor Linux.

O servidor Linux foi configurado utilizando o Linux RedHat 5.1, sendo responsável pela disponibilização de imagens para usuários externos à rede do Hospital. Nesse caso, por questões de segurança, o usuário deve solicitar as imagens desejadas, que serão disponibilizadas em uma área específica no servidor para "download" através de FTP (FTP é um protocolo que permite a transferência de um ou mais arquivos via "internet", por meio de um "browser" de navegação ou de um programa específico). O servidor FTP do CCIFM possibilita conexões restritas e anônimas. Na sua configuração atual é necessária a utilização de programas específicos, estando prevista a abertura de uma área para FTP via "browser".

Para as imagens obtidas pelo sistema de captura de vídeo a visibilização pode ser feita por meio de programas convencionais de manipulação como o PhotoShopPro-JASC ou o Adobe PhotoshopAdobe, por exemplo. Para as imagens em formato DICOM 3 a visibilização necessita de programas DICOM compatíveis como o Magic View 300 ou o Osiris, por exemplo, que é um programa livre que pode ser obtido pela rede ${ }^{(24)}$. As possibilidades de manipulação das imagens são bastante diferentes nos dois casos. As imagens capturadas permitem o ajuste de brilho, contraste e a aplicação de processamentos padrões como realce de bordas, binarização, borramento e outros. As imagens em formato DICOM 3 possibilitam processamentos mais sofisticados como mudança de janela de visibilização, remoção de caracteres alfanuméricos, realização de medidas e outras funções. Nesse caso, as imagens podem ser manipuladas da mesma forma que no console do aparelho.

\section{DISCUSSÃO}

Embora reconhecida como centro de excelência e referência na área da saúde, a FMRP-USP esteve, durante anos, praticamente à margem dos benefícios que o avanço tecnológico vem proporcionando em outras instituições. O CCIFM do Departamento de Clínica Médica vem trabalhando para modificar esta situação, buscando o desenvolvimento de conhecimentos tecnológicos e a formação de recursos humanos, de modo a suprir a demanda existente nessa área. Nesse contexto, a implantação de um serviço de radiologia "filmless" deverá trazer melhorias no que se refere à acessibilidade e integração de informações, pela vinculação de imagens ao registro médico eletrônico do paciente, e no que se refere à aplicação de novas técnicas e desenvolvimentos na aquisição, exibição e processamento de imagens. Embora de forma ainda não automatizada, a atual configuração do mini-PACS possibilita o armazenamento e a disponibilização de imagens de TC e RM, dentro e fora do ambiente hospitalar, por meio de rede "internet" utilizando protocolos TCP/IP e FTP. Foi iniciado o desenvolvimento de uma interface que permitirá a busca e visibilização de imagens a partir do registro eletrônico do paciente e está em processo de estudo a conexão de equipamentos de ultra-som à rede do miniPACS.

\section{REFERÊNCIAS}

1. Siegel EL, Reiner BI. Challenges associated with the incorporation of digital radiography into a picture archival and communication system. J Digit Imaging 1999;12(2 suppl. 1):6-8.

2. Sonoda M, Takano M, Miyahara J, Kato H. Computed radiography utilizing scanning laser stimulated luminescence. Radiology 1983;148:833-8.

3. Cannavo M. Components of PACS. In: Baumann R, ed. Understanding PACS - handbook of SCAR. Great Falls, VA: SCAR, 1992:13-8.

4. Siegel EL, Kolodner RM. Filmless radiology: state of the art and future trends. In: Siegel EL, Kolodner RM, eds. Filmless radiology. Baltimore: Springer-Verlag, 1999:3-20,177,208

5. Tucker DM, Barnes GT, Koehler RE. Picture archiving communication systems in the intensive care unit. Radiology 1995;196:297-304.

6. Junck KL, Berland LL, Bernreuter WK, McEachern M, Grandhi S, Lewey G. PACS and CR implementation in a level I trauma center emergency department. J Digit Imaging 1998;11(3 suppl. 1):159-62.

7. Allman R, Kilcoyne R, Shannon R. The PACS attraction. In: Baumann R, ed. Understanding PACS
- handbook of SCAR. Great Falls, VA: SCAR, 1992:5-8.

8. Gitlin JN. The PACS vision. In: Baumann R, ed. Understanding PACS - handbook of SCAR. Great Falls, VA: SCAR, 1992:1-3.

9. Hindel R. Systems and subsystems. In: Baumann R, ed. Understanding PACS - handbook of SCAR. Great Falls, VA: SCAR, 1992:9-12.

10. Carrino JA, Unkel PJ, Shelton P, Johnson TG. Process reengineering: the role of a planning methodology and picture archiving and communications system team building. J Digit Imaging 1999;12 (2 suppl. 1):28-31.

11. King BF Jr, Erickson BJ, Williamson B Jr, et al. Electronic imaging and clinical implementation: work group approach at Mayo Clinic, Rochester. J Digit Imaging 1999;12(2 suppl. 1):32-6.

12. Beird LC. The importance of a picture archiving and communications system (PACS) manager for largescale PACS installations. J Digit Imaging 1999;12 (2 suppl. 1):37.

13. Freeh M, Baune D. The process of converting to a near filmless operation at the University of Utah, Department of Radiology. J Digit Imaging 1999; 12(2 suppl. 1):41-6.

14. Osada M, Nishihara E. Implementation and evaluation of workflow based on hospital information system/radiology information system/picture archiving and communications system. J Digit Imaging 1999;12(2 suppl.1):103-5.

15. Ernst R, Le VT, Kawashima A, et al. A picture archiving and communications system featuring multiple monitors using Windows 98. J Digit Imaging 1999;12(2 suppl. 1):106-8.

16. Bennett WF, Spigos DG, Tzalonikou MT, Terrell JE, Augustyn MA. Web-based viewing of picture archiving and communications systems images Part I: optimal personal computer configuration. J Digit Imaging 1999;12(2 suppl. 1):112-5.

17. Bennett WF, Spigos DG, Tzalonikou MT, Terell JE, Augustyn MA. Web-based viewing of picture archiving and communications systems images Part II: the effect of compression on speed of transmission. J Digit Imaging 1999;12:(2 suppl. 1): 116-8.

18. Wendt GJ. Using commercially available off-theshelf software and hardware to develop an intranetbased hypertext markup language teaching file. J Digit Imaging 1999;12(2 suppl. 1):122-3.

19. Carbajal R, Honea R. Branching out with filmless radiology. J Digit Imaging 1999;12(2 suppl. 1): 134-6.

20. Foord K, Tomlinson N. The Conquest Hospital picture archiving and communications system development, 1992 to 1999 . J Digit Imaging 1999;12(2 suppl. 1):211-3.

21. Smith EM, Wandtke J, Robinson A. Integration, acceptance testing, and clinical operation of the medical information, communication and archive system, phase II. J Digit Imaging 1999;12(2 suppl. 1):144-7

22. Breant CM, Tiara RK, Huang HK. Interfacing aspects between the picture archiving communications systems, radiology information systems, and hospital information systems. J Digit Imaging 1993;6:88-94.

23. Azevedo-Marques PM, Santos AC, Elias Júnior J, Goes WM, Castro CR, Trad CS. Implantação de um sistema de informação em radiologia (RIS) em hospital universitário. Radiol Bras (aceito para publicação)

24. http://www.expasy.ch/UIN/html1/projects/osiris/ osiris.html. 